\title{
Molecular Electronics - Resonant Transport through Single Molecules
}

\author{
Emanuel Lörtscher* and Heike Riel
}

\begin{abstract}
The mechanically controllable break-junction technique (MCBJ) enables us to investigate charge transport through an individually contacted and addressed molecule in ultra-high vacuum (UHV) environment at variable temperature ranging from room temperature down to $4 \mathrm{~K}$. Using a statistical measurement and analysis approach, we acquire current-voltage (I-V) characteristics during the repeated formation, manipulation, and breaking of a molecular junction. At low temperatures, voltages accessing the first molecular orbitals in resonance can be applied, providing spectroscopic information about the junction's energy landscape, in particular about the molecular level alignment in respect to the Fermi energy of the electrodes. Thereby, we can investigate the non-linear transport properties of various types of functional molecules and explore their potential use as functional building blocks for future nano-electronics. An example will be given by the reversible and controllable switching between two distinct conductive states of a single molecule. As a proof-of-principle for functional molecular devices, a single-molecule memory element will be demonstrated.
\end{abstract}

Keywords: Electron transport · Mechanically controllable break-junction · Molecular building blocks . Molecular electronics $\cdot$ Single molecules



Emanuel Lörtscher holds a master in physics from the Swiss Federal Institute of Technology Zurich (ETHZ) and a PhD from the University of Basel. $\mathrm{He}$ is currently Research Staff Member at the IBM Research - Zurich Laboratory. He has gathered experience in the field of organic electronics, molecular electronics and semiconducting nanowires. His expertise includes electrical and optical characterization of semiconductor nanowires, device fabrication using e-beam lithography and charge transport measurements through single molecules. Dr. Lörtscher received the Faculty prize of the University of Basel in 2007 and the Swiss Physical Society Award for Applied Physics in the same year.

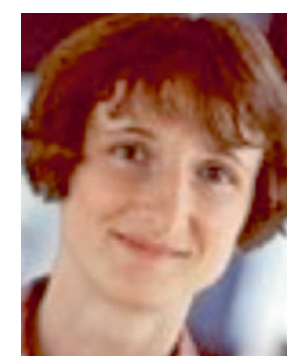

Heike Riel received her diploma in physics from the University of ErlangenNuremberg, Germany, and the $\mathrm{PhD}$ degree from the University of Bayreuth, Germany, for her work on the optimization of multilayer organic light-emitting devices. She is currently Manager of the Nanoscale Electronics Group at IBM Research - Zurich, focusing on research in the field of post-CMOS devices, in particular on the science and technology of semiconducting nanowires and molecular electronics for logic applications. Her research for both degrees was performed at IBM Research - Zurich. Her expertise includes electrical and optical characterization of organic and inorganic semiconductor materials and devices. Dr. Riel was elected to TR100, the annual list of the world's 100 Top Young Innovators by Technology Review, MIT's Magazine of Innovation and received the Award for Applied Physics of the Swiss Physical Society in 2005.

\section{Introduction}

Molecular electronics is aimed at the use of small ensembles or even individual molecules as functional building blocks in electronic circuits. ${ }^{[1]}$ Therefore, the far reaching goal is to provide various electronic functionalities by specific molecular designs, implemented and integrated into molecular device structures. One basic prerequisite to design and to build these novel types of devices is to gain a profound understanding of the relationship between chemical structure and electronic functionality. Deep insight can be gained by studying transport through ensembles of molecules, e.g. monolayers of molecules sandwiched between two electrodes, ${ }^{[2]}$ or by establishing single-molecule junctions by using, e.g. the Scanning Tunneling Microscope (STM) ${ }^{[3]}$ or mechanically controllable break-junction $(\mathrm{MCBJ})^{[4]}$ techniques. The first approach, where thousands of molecules are contacted in parallel, revealed intriguing features such as negative differential resistance, ${ }^{[5]}$ rectification behavior, ${ }^{[6]}$ Kondo effect, ${ }^{[7]}$ or conductance switching. ${ }^{[8]}$ However, in order to avoid ensemble effects, measurements on the single-molecule level are important. Hereby it was revealed that transport can be predominated by the molecule-metal contact which is prone to contact variations due to variable bonding sites (e.g. various possible configurations of the sulfur-gold ( $\mathrm{S}-\mathrm{Au})$ bond) or weak electronic overlap. ${ }^{[5,9]}$

In this article, we describe a statistical measurement approach which provides spectroscopic information on the metalmolecule-metal junction by the acquisition of current-voltage (I-V) characteristics while mechanically manipulating the microscopic configuration of the molecular junction. ${ }^{9]}$ Examples for resonant transport will be given by measurements through simple conjugated building blocks such as phenyl-dithiol[9] or a series of oligophenylenes synthesized to study the 
length dependence. ${ }^{[10]}$ Furthermore, functional molecules were studied, revealing a voltage-induced switching between two distinct conductive states which can be exploited to demonstrate memory operation on a single-molecule level. ${ }^{[11]}$

\section{Mechanically Controllable Break- junction Technique}

All the charge transport studies reported in this contribution are conducted using the MCBJ technique[12] (Fig. 1). This approach has been proven to enable charge transport measurements through an individual molecule. ${ }^{[13]}$ Our MCBJ system is operated under ultra-high-vacuum (UHV) conditions in the temperature range between 4 and $300 \mathrm{~K}$ (see refs [9-11] for further experimental details). Electronbeam lithography and reactive ion etching are used to fabricate a freestanding metal bridge on top of a flexible substrate (Fig. 1a). The initial cross-section of the $\mathrm{Au}$ bridge, typically $50 \mathrm{~nm} \times 50 \mathrm{~nm}$, is continuously reduced upon bending of the substrate in a three-point bending mechanism (Fig. 2b). Just before breaking, the crosssection of the bridge is reduced to a single $\mathrm{Au}$ atom. Such a quantum point contact (QPC) is indicated by the appearance of quantized conductance with multiple integer values of the conductance quantum, $\mathrm{G}_{0}$ $\left(\mathrm{G}_{0}=2 \mathrm{e}^{2} / \mathrm{h} \sim 1 / 12.9 \mathrm{k} \Omega\right)$. Further stretching finally breaks the bridge and creates thereby two atomic-sized electrodes opposite to each other.

After breaking, the distance between both electrodes can be controlled by the bending of the substrate, with a very high accuracy due to the low transmission ratio between pushing rod translation, $\Delta \mathrm{h}$, and resulting electrode separation, $\Delta \mathrm{d}$, in the order of $10^{-5}$. Consequently, the resolution can be sub-atomic and a high lateral stability is achieved due to the close proximity of the supports $(\mathrm{s}<1 \mu \mathrm{m})$. Before applying molecules, the electrodes are shaped in a conditioning procedure until reproducible plateaus at (1-5) $\times \mathrm{G}_{0}$ are measured, indicating atomic-sized electrodes.

For the application of molecules, the distance between the electrodes is then set to be longer than the length of the molecule to be studied (a calibration of the actual electrode displacement is made by analyzing $50-500$ closing traces and fitting them to an exponential electron tunneling decay). The molecules are then deposited from a highly diluted solution (typically $4 \times 10^{-5} \mathrm{~mol} / \mathrm{L}$ ) and can hereby adsorb to one of the two electrode surfaces while the solvent evaporates. Subsequently, the system is evacuated and measurements are performed when reaching ultra-high vacuum conditions (pressure

\section{a Electric Circuit}

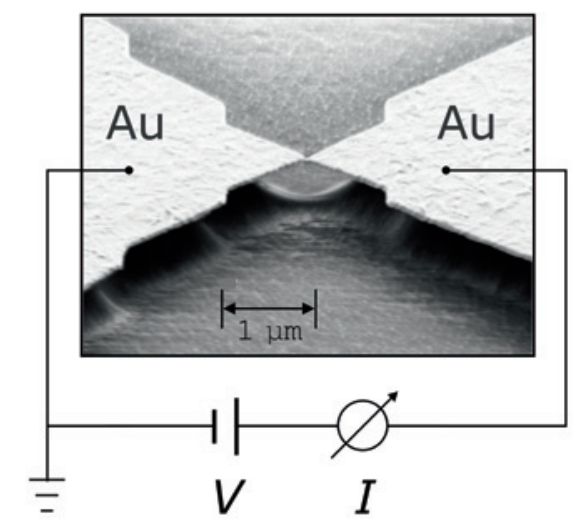

b MCBJ Principle

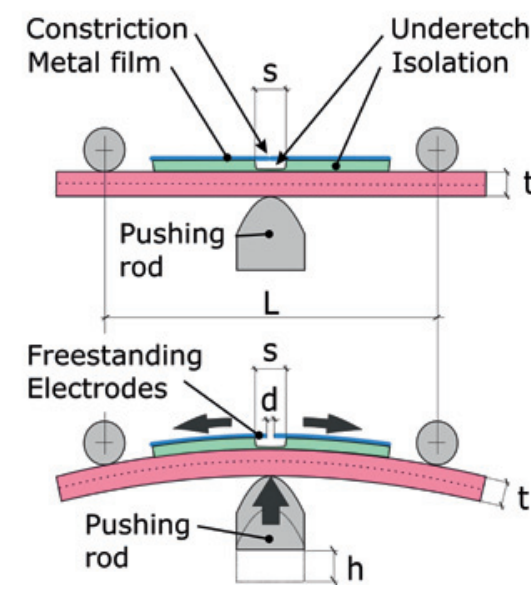

Fig. 1. a) Scanning electron microscopy (SEM) picture of a freestanding metal bridge fabricated by electron-beam lithography on top of a flexible and insulating substrate. The electrical circuit, representing the two-terminal geometry, is illustrated schematically. b) The bending of the substrate in a three-point bending mechanism c) stretches and finally breaks the bridge at its smallest constriction, creating thereby two separated electrodes. Reprinted with permission from ref. [11a]. Copyright Wiley-VCH Verlag GmbH \& Co. KGaA.

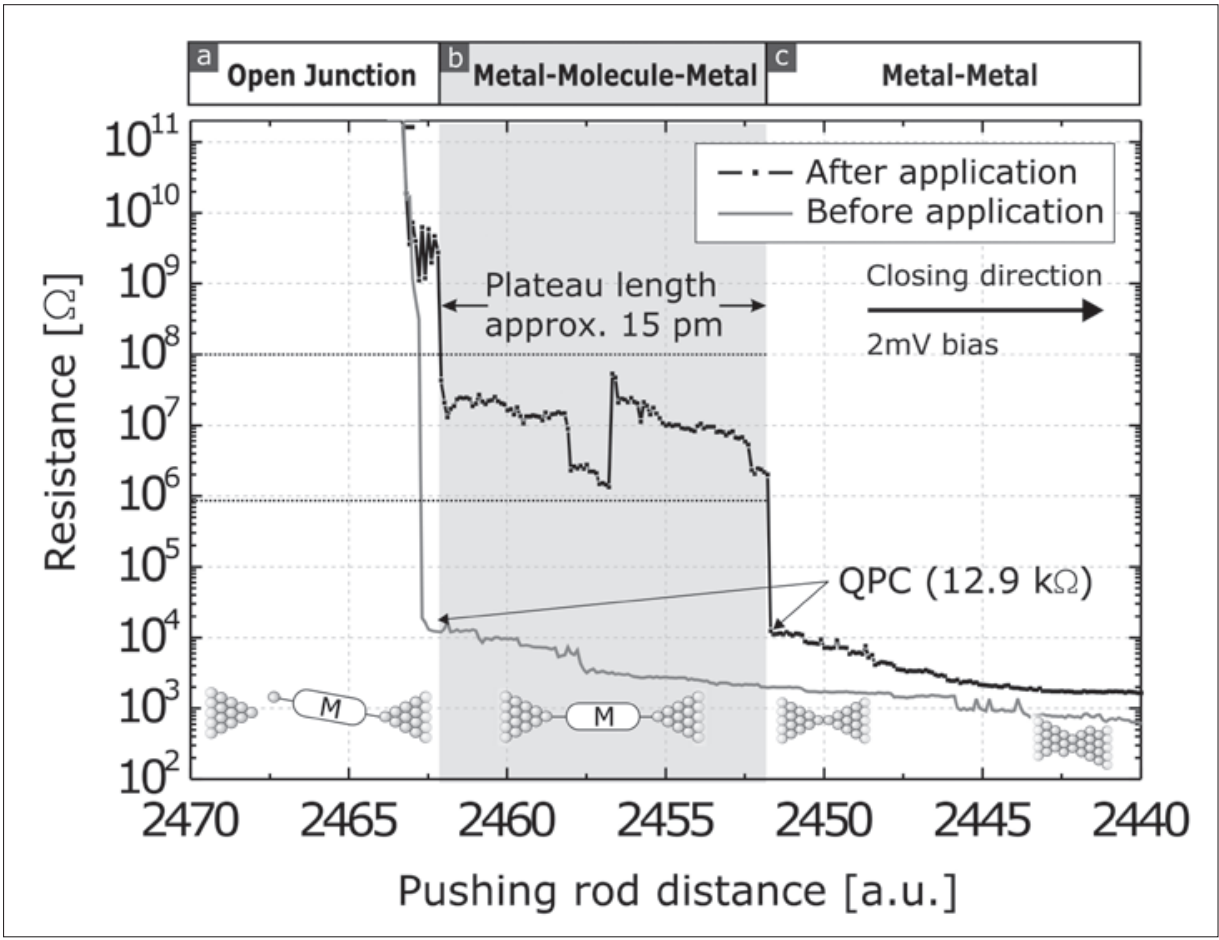

Fig. 2. Closing of a molecular junction under an applied small bias of $2 \mathrm{mV}$ : a) Open junction with a resistance, $\mathrm{R}>1 \mathrm{~T} \Omega$, b) formation of metal-molecule-metal junction, and c) closed metalmetal point contact (QPC) with $\mathrm{R}<12.9 \mathrm{k} \Omega$. The gray curve shows the closing behavior before application of molecules without the appearance of an intermediate regime. Reprinted with permission from ref. [11a]. Copyright Wiley-VCH Verlag GmbH \& Co. KGaA.

$\mathrm{p}<10^{-8}$ mbar). UHV environment is a prerequisite to preserve the integrity of the molecular system from unwanted contamination by hydrogen, oxygen or other reagents. During the subsequent closing of the junction, molecules can bridge the gap between the electrodes by establishing the second chemical bond to the counter electrode, thus forming a molecular junction. This formation can be observed in resistance vs. electrode distance plots, when resistance plateaus appear (Fig. 2) in the intermediate regime between an open junction $(\mathrm{R}>1 \mathrm{~T} \Omega)$ and a closed $\mathrm{Au}-\mathrm{Au}$ junction $(\mathrm{R}<12.9 \mathrm{k} \Omega)$. In this plateau regime, the transport properties of metal-molecule-metal junctions can be characterized and studied in detail. 


\section{Statistical Approach}

In single-molecule junctions, the molecule as the functional building block itself is comparable in size with the atoms composing the electrodes. Since the position of these atoms cannot be controlled with sub-atomic accuracy (especially at room temperature), the actual microscopic configuration of the junction depends on the gap distance, the geometric shape of the electrodes, the resulting electrical field distribution, and the specific bonding of the molecule-metal interfaces. As a consequence, all transport measurements have to be carried out statistically taking these considerations into account. Therefore, usually several hundreds or thousands of closing and opening curves are measured under at small constant bias during formation and breaking of the molecular junction. ${ }^{[3]}$ Often these comprehensive data sets acquired are filtered using artificial data selection schemes, and statistically analyzed, by creating histograms or breaking-distance plots. Thereby, single-molecule conductance values can be extracted as schematically illustrated in Fig 3a. ${ }^{[9]}$

These low-bias measurements reveal the transport properties in a non-resonant tunneling regime because for most of the organic molecules used in the field of molecular electronics, the Fermi energy of $\mathrm{Au}$, $\mathrm{E}_{\mathrm{F}, \mathrm{Au}}$, lies somewhere between the highest occupied molecular orbital (HOMO) and the lowest unoccupied molecular orbital (LUMO) of the isolated molecule. Upon coupling the molecule to the electrodes, a charge transfer from the molecule to the metal (or vice versa) occurs, shifting the levels and broadening them because of hybridization between molecular states and metal wave functions. Even though the amount of charge can be substantial for strong coupling, the orbitals are still wellseparated from $\mathrm{E}_{\mathrm{F}, \mathrm{Au}}$. As a consequence, the low-bias measurements probe the very low density of states (DOS) in the HOMOLUMO gap of the molecule.

In order to get an insight into transport properties and molecular functionality and to gain an understanding of the structure/ function relationship, it is essential to study the transport properties over a voltage range where we can probe the first molecular states (typically $0.5-3.0 \mathrm{~V}$ ). This enables us to monitor and to determine the characteristic signatures of the molecule under investigation in the resonant regime ${ }^{[9]}$ and to gather spectroscopic information about the junction's electronic configuration. This is a basic requirement to understand the non-linear transport properties on the molecular level. Hence, we continuously acquire I-V curves during stepwise closing and opening, corresponding to different microscopic configurations

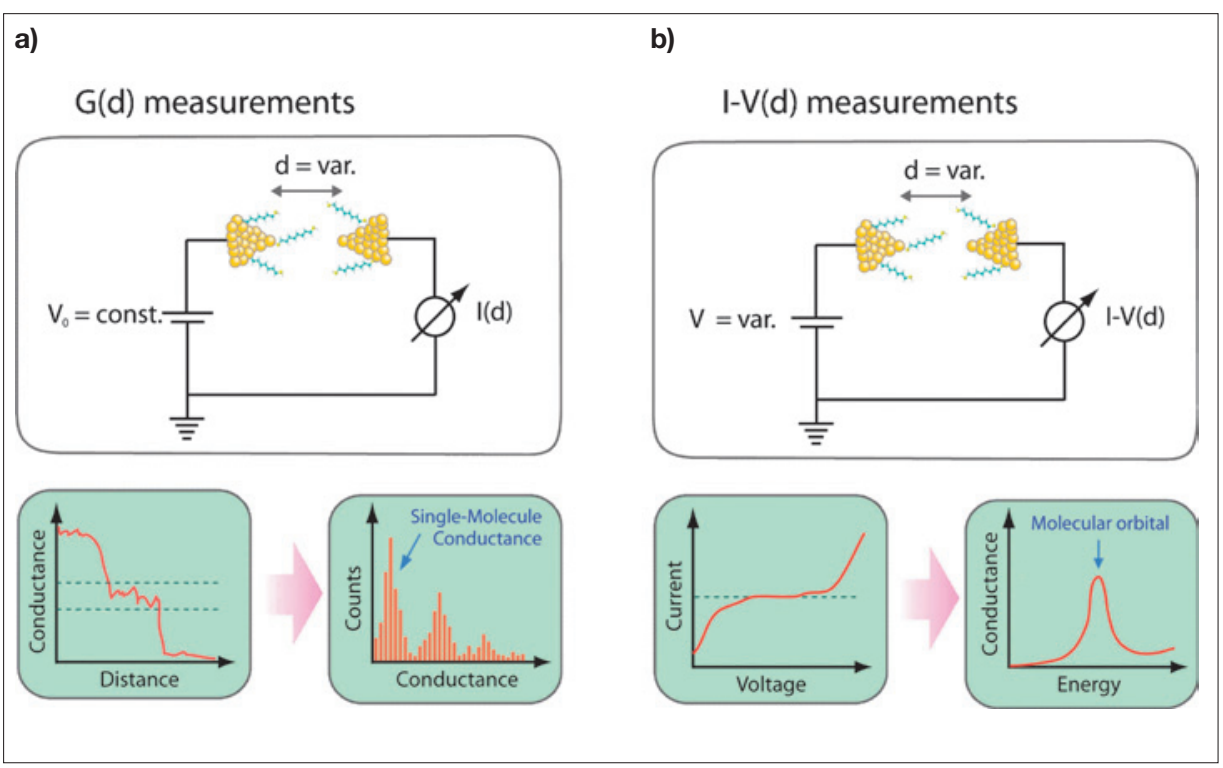

Fig. 3. a) Transport measurements acquired under a small bias $(\mathrm{V}<100 \mathrm{mV})$ while varying the electrode gap distance result in individual conductance-distance $G(d)$ curves. Histograms are established from a multitude of $\mathrm{G}(\mathrm{d})$ curves. Peaks at distinct conductance values may be referred to the single-molecule conductance. b) Identical electrode driving protocol as in a) but with stepwise stopping and measurement of an entire I-V curve. This procedure allows resonant transport through molecular orbitals to be conducted and spectroscopic information about the energy landscape of the molecular junction to be acquired.

of the molecular junction. This approach is illustrated schematically in Fig. $3 \mathrm{~b}$ and described below and in ref. [9].

After application of the molecules as described above, the molecules are attached to one electrode only and a current between $1 \mathrm{fA}$ and $1 \mathrm{pA}$ is measured, indicating the noise-level of an open junction without molecules bridging the gap. When decreasing the distance between the electrodes, an abrupt jump to stable and reproducible I-V curves is observed signaling the formation of the molecular junction. Now, the current flow is in the nanoampere (nA) range and the I-V curves exhibit a nonlinear behavior, which shows the characteristic signature of the particular metal-molecule-metal system under investigation. ${ }^{[9]}$ During further closing, the $\mathrm{I}-\mathrm{V}$ characteristics are maintained over a range that is in good agreement with the length of the intermediate regime observed during closing or opening under a constant bias (Fig. 2b). Beyond this region, a sudden jump to much higher currents in the microampere $(\mu \mathrm{A})$ range occurs where the current shows a linear behaviour, indicating a closed metal-metal junction. At this stage, the direction of electrode motion is reversed and a subsequent opening cycle is performed accordingly. This closing-opening procedure is repeated several dozens of times. Owing to ductilityinduced drifts in the electrodes, we control the junction via the resistance measured rather than via $\Delta \mathrm{h}$. This automated measurement procedure provides objectivity to the investigations, and the entity of all data represents a reliable basis for statistical analysis. To determine, for example, the number of molecules captured in the junction, conductance histograms at any arbitrary voltage can be extracted from huge data sets. Thereby, the most probable $\mathrm{I}-\mathrm{V}$ characteristics in the ensemble of all measured curves can be identified.

As an example for the sensitivity of our approach to sub-1nm long molecules, we present data on the benzene-1,4-dithiol (BDT). This prototypical molecule is of fundamental interest as it is one of the simplest molecules having a conjugated system that is strongly coupled via two tunneling barriers to metallic leads. Pioneering experimental work was conducted by Reed et al., ${ }^{[14]}$ using the MCBJ technique with a manually notched wire covered by a self-assembled monolayer of BDT molecules. This work triggered many theoretical investigations. ${ }^{[15]}$ However, depending on the various approximations and models, the calculated conductance values often differ from the experimental data. In our measurement, the Au-BDT-Au system is cooled down to low temperatures after having reached the intermediate regime of the closing trace at room temperature. This procedure causes the electrodes to be withdrawn due to thermal compression $\Delta \mathrm{d}$ $\sim 5-10 \mathrm{~nm}$ for $\Delta \mathrm{T} \sim 250 \mathrm{~K}$, resulting in a breaking of the molecular junction. Now, starting again with an open junction, I-V curves are acquired using the statistical approach. Fig. 4a shows a set of $\mathrm{I}-\mathrm{V}$ raw data 
curves for BDT taken at $250 \mathrm{~K}$ representing several dozens of curves acquired at each electrode position. The slight asymmetry of the individual I-V curves disappears when plotting all curves measured in the regime of cumulative occurrence and creating their envelope (gray area). Between 100 and $250 \mathrm{~K}$, the Au-BDT-Au junction can be opened and reformed for many cycles. In contrast, at $50 \mathrm{~K}$ (Fig. $4 b$ ), data acquisition is only possible for a few cycles at each position because the junction becomes instable which is characteristic for sub-1nm long molecules. For both data sets, the differential conductance $\left(\mathrm{G}_{\text {Diff }}\right)$ vs voltage plot shows two peaks. At $250 \mathrm{~K}$, they are located at approximately $-0.5 \mathrm{~V}$ and $+0.4 \mathrm{~V}$, defining a conductance gap $2 \cdot \Delta_{\text {cond }}$ of $0.9 \mathrm{~V}$. At 50 $\mathrm{K}$ (Fig. 4b), $2 \cdot \Delta_{\text {cond }}$ is on the order of 0.6 $\mathrm{V}$. Such a significant shift of $300 \mathrm{mV}$ for $\Delta \mathrm{T}=200 \mathrm{~K}$ cannot solely be explained by freeze-out of vibrational modes. Instead, a different charge transfer from the metal to the molecule resulting in an altered charging energy depending on temperature can shift the resonant molecular energy levels and thus the conductance gap. Furthermore, different atomistic configurations of the molecule-metal contact at various temperatures can also lead to such an effect. In Fig. 4b, the current signal above $+0.6 \mathrm{~V}$ and below $-0.6 \mathrm{~V}$ reveals significantly more noise, which is inherent to the metal-BDT-metal system. This is found to be very similar to the data presented in ref. [14] for $\mathrm{V} \pm 3.0 \mathrm{~V}$. At $300 \mathrm{~K}$, in contrast to ref. [14], we were not able to establish a metal-BDT-metal junction prior to a metal-metal contact when performing I-V curves, even for samples that formed molecular junctions at lower temperatures. This could be due to the mentioned ductile response to deformation of the gold electrodes, which is more pronounced at higher temperatures and exceeds obviously a critical value for the sub-nanometer-long BDT (S-S distance: $0.627 \mathrm{~nm}$ ) under such high electric fields of about $1.4 \times 10^{9} \mathrm{~V} / \mathrm{m}$.

Though on the first sight, our findings look similar to ref. [14], several strong differences should be stressed: First, the voltage scale is substantially reduced, with a conductance gap of $0.3 \mathrm{~V}(1.4 \mathrm{~V}$ in ref. [14]), and a stability region limited to $\pm 1.2 \mathrm{~V}( \pm 5 \mathrm{~V}$ in ref. [14]). No further steps in the differential conductance were observed within the voltage window available. The conductance values beyond the first current step, although recorded at different temperatures, are rather similar, 70 $\mathrm{nS}$ compared to $45 \mathrm{nS}$. ${ }^{[14]}$ Compared to theoretical results both experimental values are 2 to 3 orders of magnitude lower. However, the overall shape of the I-V characteristics reveals a pronounced conductance gap and fluctuations for a certain

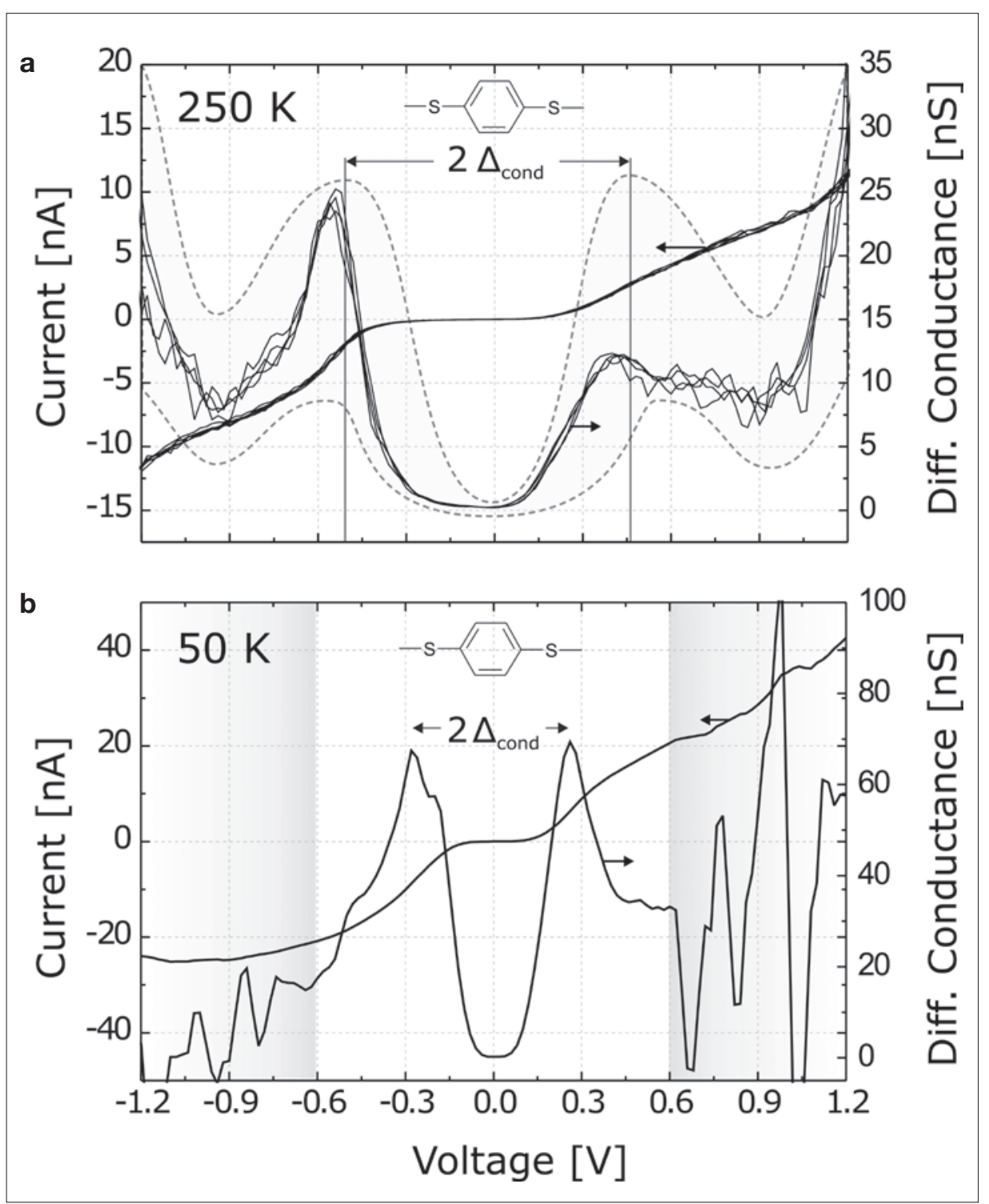

Fig. 4. $I-V$ and $G_{D i f f}-V$ characteristics of BDT measured at (a) $250 \mathrm{~K}$ and (b) $50 \mathrm{~K}$. The gray area limited by the dotted line in (a) shows the envelope function of all curves measured. Reprinted with permission from ref. [9].

threshold voltage ${ }^{[14]}$ similar to the experimental results.

Low-bias measurements were performed for BDT as well, showing a resistance peak at around $(260 \pm 20) \mathrm{M} \Omega .{ }^{[9]}$ Compared to the resonant resistance value of $(14 \pm 4) \mathrm{M} \Omega$, it becomes obvious that even for a simple conjugated system coupled via thiol to $\mathrm{Au}$, the contact resistance of the molecule-metal interface is the predominant factor in the junction's resistance which renders molecular junctions rather insulators than good conductors. This fact clearly signals the need for an electronically more efficient anchor group than thiols for future nano-electronic applications using single-molecule building blocks. This experimental background raises the question whether changes in the intramolecular structure of single molecules are detectable in the transport properties or whether they are masked by the contact resistance.

\section{Influence of Molecular Length}

The influence of molecular length on the transport properties of single molecules has been studied extensively for alkanes ${ }^{[16]}$ using the histogram approach as described before. Thus, primarily the off-resonance transport properties at fixed low bias were derived. Only a few systematic studies of the influence of the molecule length on the resonant transport properties exist. ${ }^{[17]}$

We have investigated the length dependence on charge transport for a series of oligophenylene rigid rod-like molecules by measuring $\mathrm{I}-\mathrm{V}$ characteristics in the resonant regime. Methyl groups are attached to the phenyl rings to weaken the electronic overlap of the $\pi$-subsystems along the molecular backbones (Fig. 5a). Out-of-plane rotation of the phenyl rings was confirmed in the solid state by means of X-ray analysis and in solution by using UV/Vis spectroscopy. ${ }^{[10]}$ A linear increase 
of the conductance gap with increasing number of phenyl rings (from $260 \mathrm{meV}$ for one ring to $580 \mathrm{meV}$ for four rings) was revealed (Fig. 5c). In addition, the absolute conductance of the first resonant peak for each molecule does not directly depend on the length of the molecular wire (Fig. 5b). Hence, resonant transport through the first molecular orbital was found to be dominated by charge-carrier injection, rather than by the intrinsic resistance of the molecular wire length. ${ }^{[10]}$ Remarkably, the spectroscopic data of molecules which possess a structural degree of freedom to change conformation, show insensitive conductance peaks upon mechanical manipulation of the gap distance, e.g. for a cardan-joint like molecule. ${ }^{[18]}$ Thereby, the fluctuations in the cardan angle leave the positions of steps in the I-V curve largely invariant. ${ }^{[18]}$ As a consequence, the experimental I-V characteristics exhibit LUMO-based conductance peaks at particular voltages, which are also found to be temperature independent. To summarize, even though the contact resistance dominates the transport properties, changes in the intramolecular structure are revealed in the transport measurements.

\section{Intrinsic Functionality - Switching and Memory Operation}

The vision of molecular electronics is to use functional building blocks for future electronic circuits where $e . g$. the conductance can be switched 'on' and 'off', triggered by an external stimulus such as light, voltage or electrical field. We have investigated the transport properties of single bipyridyl-dinitro oligo-phenylene-ethynylene dithiol (BPDN-DT, Fig. 6a) molecules and bipyridyl oligophenylene-ethynylene dithiol (BP-DT, Fig. 6b) molecules connected to gold electrodes at $100 \mathrm{~K}$. An unipolar voltage-triggered conductance switching of an ensemble of BPDN-DT molecules was reported using three different techniques. ${ }^{[19]}$ In our experiments, the BP-DT molecule acts as a reference since it does not possess the functional nitro groups attached to the central bipyridyl units. The I-V characteristics of the two molecules exhibit the same conductance gap but with an approximately six times lower current for BPDN-DT. ${ }^{[11]}$ The lower conductance can be explained by the reduced conjugation of BPDN-DT due to its more twisted molecular structure caused by the steric hindrance of the nitro groups. Noticeable, in the I-V curve of BPDN-DT is the appearance of a hysteresis at $\pm 0.7 \mathrm{~V}$ as shown in Fig. 6c. In contrast, the BP-DT exhibits no hysteresis and exclusively monotonic traces without any switching. Stochastic

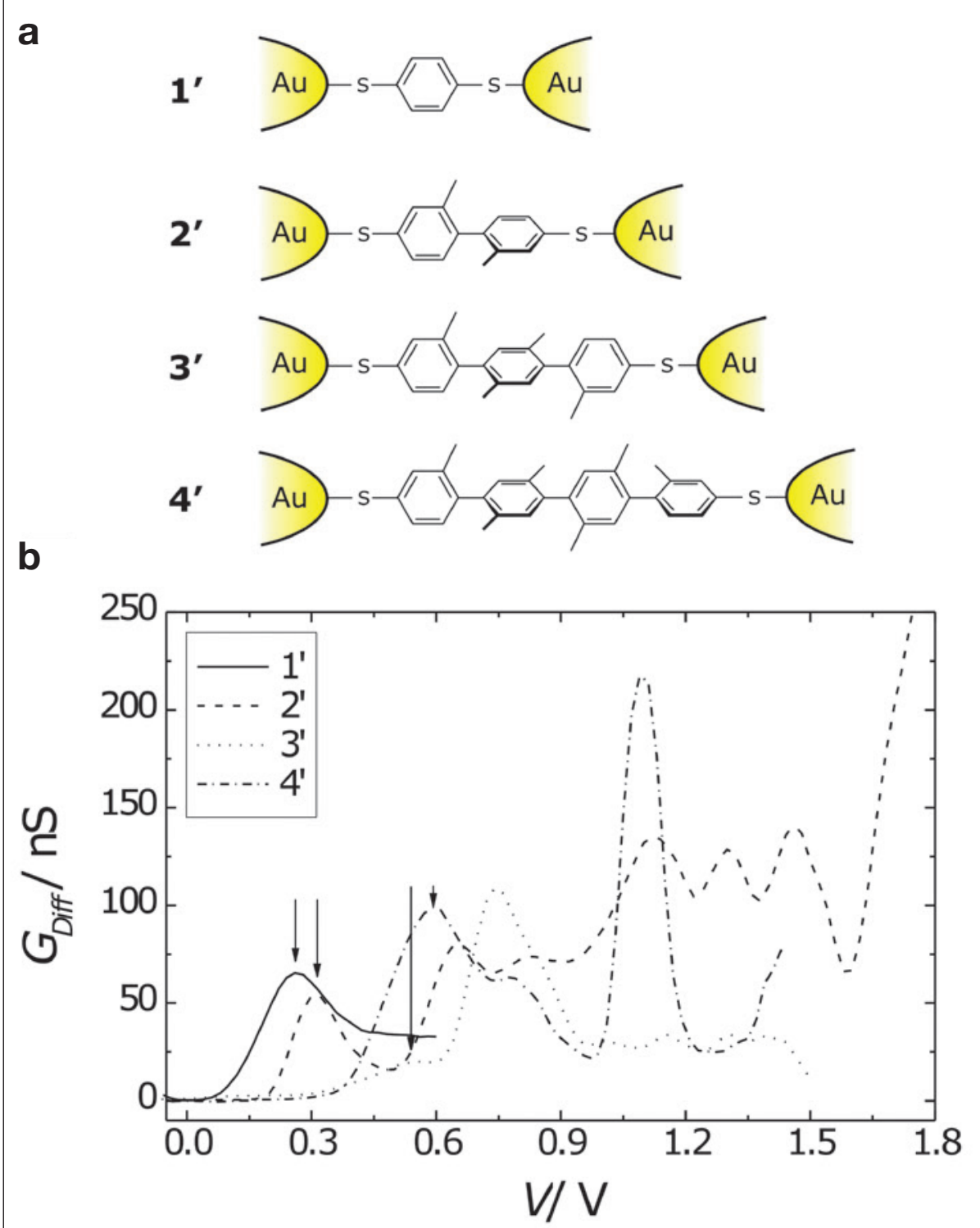

C

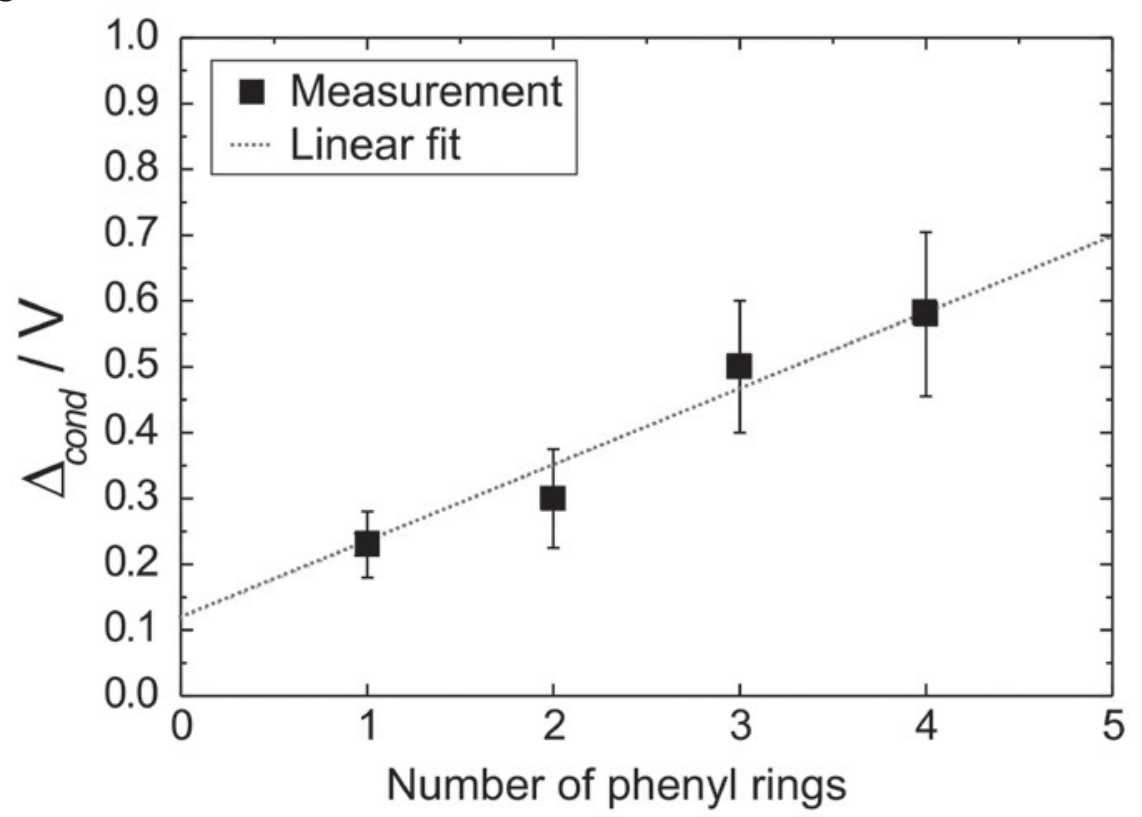

Fig. 5. a) Series of oligophenylenes with increasing length of the molecular backbone. b) Differential conductance data for the series of oligophenylenes acquired at $50 \mathrm{~K}$. c) Conductance gap, $\Delta_{\text {cond }}$, as a function of the number of phenyl rings (the error bars indicate the statistical variations among all the samples measured). Reproduced with permission from ref. [10]. 


\section{a BPDN-DT}

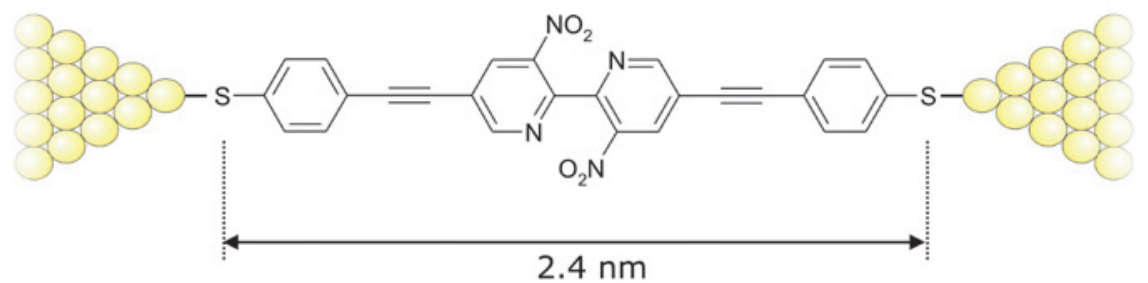

b BP-DT

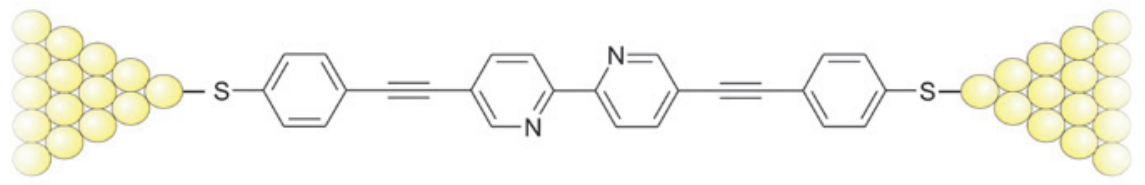

C

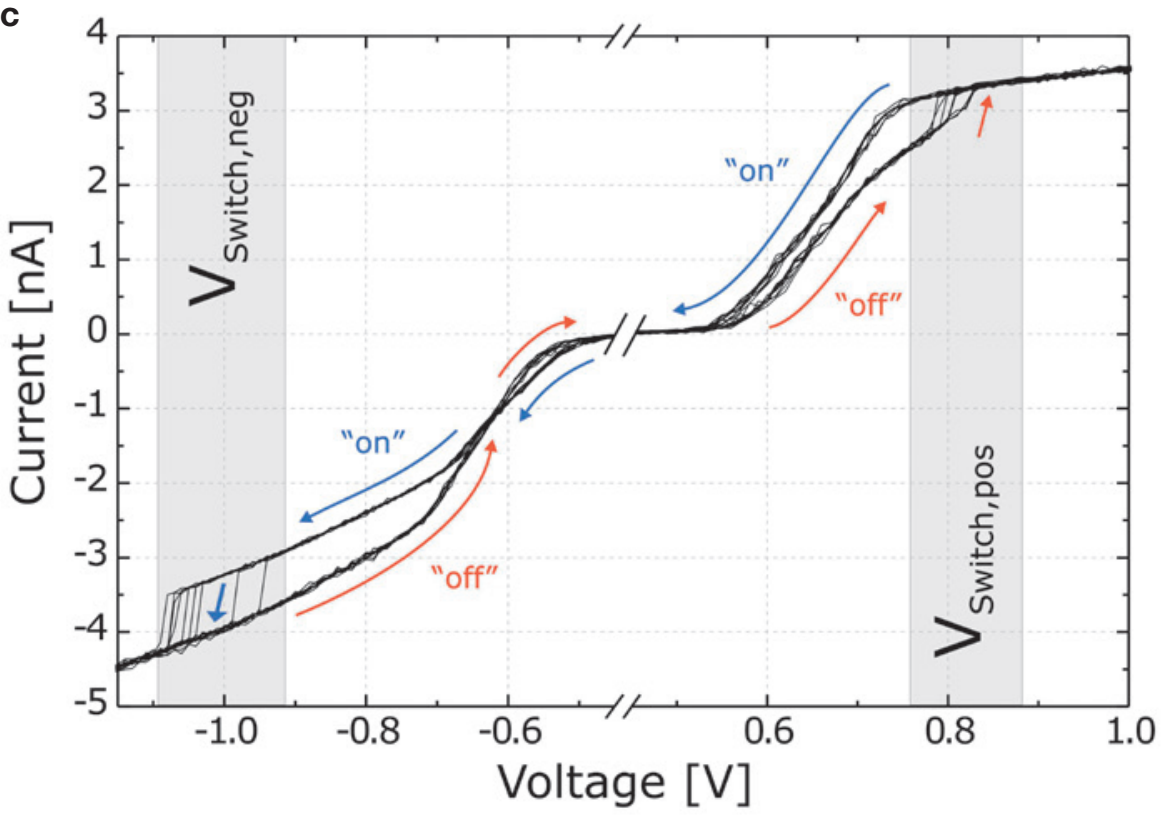

Fig. 6. a) Molecular structures of BPDNDT, and b) BP-DT molecules. c) Several repeated switching cycles of the BPDN-DT exhibiting a hysteresis. Reprinted with permission from ref. [11a]. Copyright Wiley-VCH Verlag GmbH \& Co. KGaA.

switching ${ }^{[11]}$ is absent for both molecular systems mainly due to the measurement at low temperatures (100 K). Typically we observe statistical fluctuations in the molecule itself or the molecule-metal contact only at elevated temperatures $(\mathrm{T}>250 \mathrm{~K})$. Consequently, the mechanism causing the switching observed in BPDN-DT is not of a stochastic type but has truly a molecular origin, which is caused by the functional nitro groups. [11]

The conductance switching observed for BPDN-DT is very stable and reproducible. Fig. $6 \mathrm{c}$ shows several I-V curves taken for BPDN-DT at $100 \mathrm{~K}$ revealing a hysteresis for positive and negative voltages. Starting the measurement from $0 \mathrm{~V}$, the current signal increases monotonically until the voltage exceeds $\mathrm{V}_{\text {Switch,pos }}$. The cur- rent signal suddenly jumps from a low conductive curve, called the 'off' trace, at approximately $+0.8 \mathrm{~V}$ to a high current curve, called the 'on' trace. Sweeping the voltage back, one observes a hysteresis. After this first switching sweep, when operating only at voltages above $\mathrm{V}_{\text {Switchneg }}$, the initial 'off' state is no longer accessible and the metal-molecule-metal system remains in the higher conductive 'on' state during all subsequent positive sweeps. Performing a negative voltage sweep, a similar switching behavior is observed. Sweeping back, the current signal follows again a monotonous trace. This negative sweep resets the system to the 'off' state, which is verified by a subsequent positive sweep that again exhibits a lower current and a switching to the 'on' state. In all our samples, the 'off' state is always reconfigurable by applying a negative voltage pulse below a certain threshold voltage $\left(<\mathrm{V}_{\text {Switcheg }}\right)$ Both states of the system are remarkably stable: Even after several hours of measurement and performing more than 500 sweeps iteratively, the hysteresis effect is maintained and the system retains its ability to switch.

As mentioned above, voltage pulses exceeding the threshold voltages can be applied to switch the system between the two discrete states in a very controlled and reversible manner. The 'on' state is unaffected by successive read pulses at low positive voltages $\left(+0.5 \mathrm{~V}<\mathrm{V}_{\text {Read }}<\mathrm{V}_{\text {Switch,pos }}\right)$. This non-destructive reading in combination with the controlled switching in both directions makes it possible to use this singlemolecule system as a memory element.

We demonstrate memory operation by storing one bit in this molecular building block: A write pulse turns the system to 'on', which is controlled with a subsequent read-out of the bit-state by direct probing of the current. An erase pulse resets the memory element, which is again tested by another subsequent read-out. Fig. 7a displays the pulse pattern consisting of write $(+1.6 \mathrm{~V}, 50 \mathrm{~ms}$ length) and erase $(-1.6 \mathrm{~V}, 50$ ms length) pulses with intermediate readings $(+1.1 \mathrm{~V}, 3 \mathrm{~s}$ duration) which was applied to the metal-single-molecule-metal system. The inset of Fig. 7b shows the I-V curve for the specific sample used. The current through the molecule was directly probed (Fig. 7b). During the reading times of three seconds, the current signals were constant and no decay of the state was observed. The currents measured for the 'off' state vary between 0.05 and $0.13 \mathrm{nA}$, those for the 'on' state between 0.9 and $3.6 \mathrm{nA}$, yielding a bit separation ( $\left.\mathrm{I}_{\text {on }} / \mathrm{I}_{\text {off }}\right)$ ranging between 7 and 70 . Furthermore, periodic reading of a stored bit (no voltage applied between readings) established that this single-molecule memory is non-volatile over a measurement time of several minutes at $100 \mathrm{~K}$ (see ref. [11] for more details).

\section{Conclusions}

To summarize, we have demonstrated that resonant transport measurements, performed by the statistical approach, provide spectroscopic data on the energetic level alignment of the molecule in the junction. The molecular resonant conductance values, however, are still small compared to off-resonant conductance measured at low bias and do not reach theoretical predictions. Further improvements in the molecule-metal coupling are hence strongly required by designing advanced linker groups which provide smaller contact resistance and fewer variations as originating from the distinct bonding sites of sulphur on gold. 


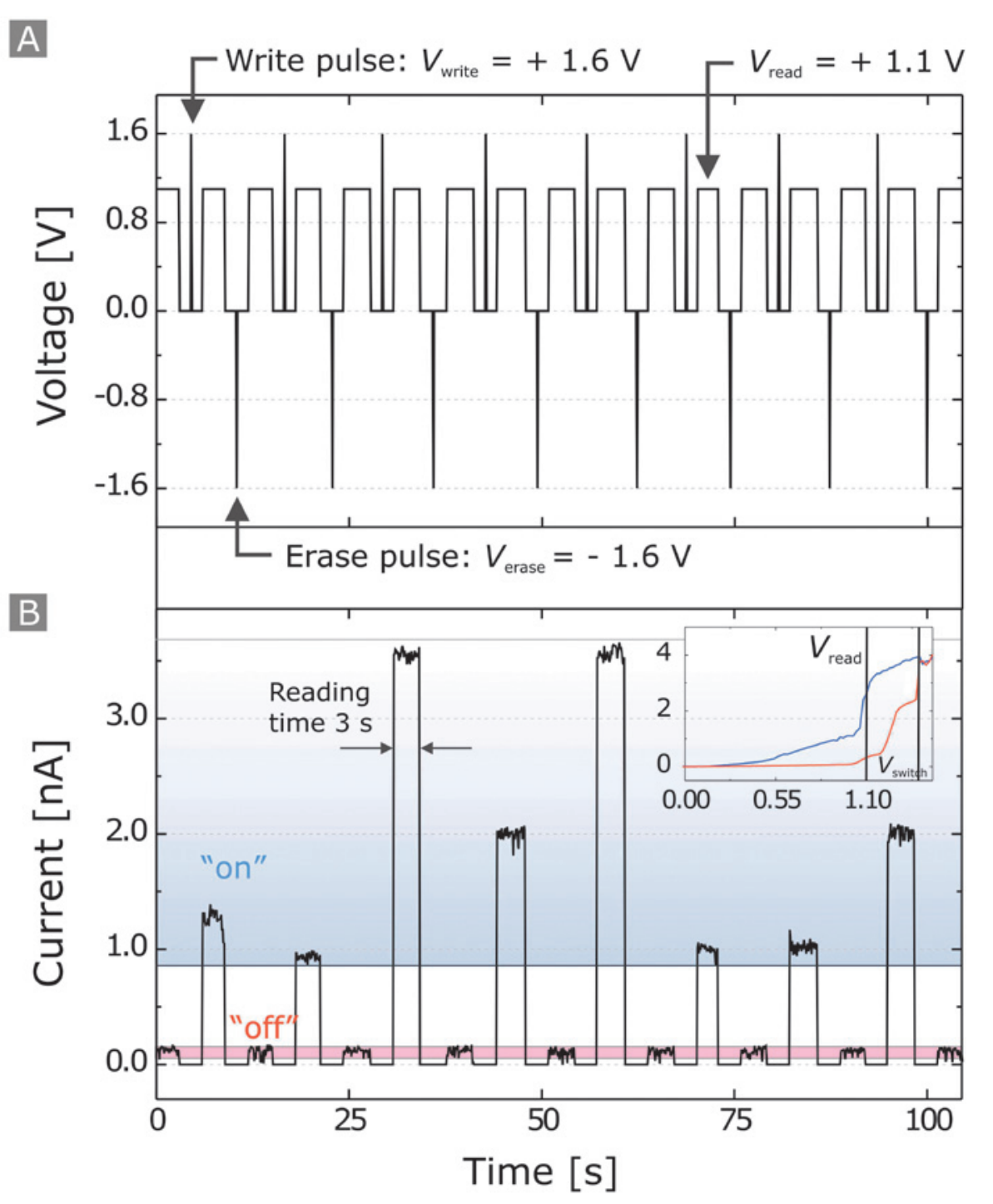

Fig. 7. a) Write, read and erase voltage pulse pattern applied to the Au-BPDN-DT-Au junction, at $100 \mathrm{~K}$. b) Current reading reveal a controlled switching between 'off' and 'on' states, demonstrating single-molecule memory operation. Reprinted with permission from ref. [11a].

The demonstration of a single molecule switch represents an important step on the path to the ultimate limit in device scaling. The switching was investigated by charge transport through an individually contacted and addressed molecule using the mechanically controllable break-junction technique and was attributed to the inherent properties of the molecule. Both conductive states can be controlled by a simple voltage pulse and are stable and accessible via non-destructive reading, enabling repeated write-read-erase-read cycles. For the first time more than 500 such cycles of bit storage in this single-molecule memory element have been demonstrated.

\section{Acknowledgements}

We gratefully acknowledge Walter Riess, Siegfried Karg and Bernd Gotsmann for many helpful discussions and Marcel Mayor and James Tour and their groups for providing molecules. Furthermore, we greatly appreciate technical support by Meinrad Tschudy and processing support by Ute Drechsler and Charles Rettner (e-beam lithography). We acknowledge the European Community's Seventh Framework Program (FP/2007-2013) and the grant agreement 'SINGLE' no. 213609.

\section{Received: April 20, 2010}

[1] a) M. A. Ratner, A. Aviram, Chem. Phys. Lett. 1976, 29, 277; b) M. Ratner, Mater. Today 2002, 5, 20; c) R. L. Carroll, C. B. Gorman, Angew. Chem. 2002, 114, 4556; d) 'Molecular Electronics: Commercial Insights, Chemistry, Devices, Architecture and Programming', Ed. N. J. Teaneck, World Scientific, Singapore, 2003; e) R. L. Carroll, C. B. Gorman, Angew. Chem. 2002, 114, 4556 .

[2] a) C. P. Collier, E. W. Wong, M. Belohradsky, F. M. Raymo, J. F. Stoddart, P. J. Kuekes, R. S. Williams, J. R. Heath, Science 1999, 285 , 391; b) J. Chen, M. A. Reed, A. M. Rawlett, J. M. Tour, Science 1999, 286, 1550; c) M. A. Reed, J. Chen, A. M. Rawlett, D. W. Price, J. M. Tour. Appl. Phys. Lett. 2001, 78, 3735; d) Y.
Luo, C. P. Collier, J. O. Jeppesen, K. A. Nielsen, E. Delonno, G. Ho, J. Perkins, H. R. Tseng, T. Yamamoto, J. F. Stoddart, J. R. Heath, Chem. Phys. Chem. 2002, 3, 519.

[3] a) Z. J. Donhauser, B. A. Mantooth, K. F. Kelly, L. A. Bumm, J. D. Monnell, J. J. Stapleton, D. W. Price, A. M. Rawlett, D. L. Allara, J. M. Tour, P. S. Weiss, Science 2001, 292, 2303; b) B. Xu, N. Tao, Science 2003, 301, 1221.

[4] J. Reichert, H. B. Weber, M. Mayor, H. v. Löhneysen, Appl. Phys. Lett. 2003, 82, 4137.

[5] a) J. Chen, M. A. Reed, A. M. Rawlett, J. M. Tour, Science 1999, 286, 1550; b) J. Chen, W. Wang, M. A. Reed, A. M. Rawlett, D. W. Price, J. M. Tour, Appl. Phys. Lett. 2000, 77, 1224.

[6] a) J. G. Kushmerick, D. B. Holt, J. C. Yang, J. Naciri, M. H. Moore, R. Sashidhar, Phys. Rev. Lett. 2002, 89, 086802; b) I. Diez-Perez, J. Hihath, Y. Lee, L. Yu, L. Adamska, M. A. Kozhushner, I. I. Oleynik, N. Tao, Nature Chemistry 2009, 1, 635 .

[7] a) H. Park, J. Park, A. K. L. Lim, E. H. Anderson, A. P. Alivisatos, P. L. McEuen, Nature 2000 , 407, 57; b) J. Park, A. N. Pasupathy, J. I Goldsmith, C. Chang, Y. Yaish, J. R. Petta, M. Rinkoski, J. P. Sethna, H. D. Abruna, P. L. McEuen, D. C. Ralph, Nature 2002, 417, 722; c) W. Liang, M. P. Shores, M. Bockrath, J. R. Long, H. Park, Nature 2002, 417, 725; d) A. N. Pasupathy, R. C. Bialczak, J. Martinek, J. E. Grose, L. A. K. Donev, P. L. McEuen, D. C. Ralph, Science 2004, 306, 86.

[8] a) M. A. Reed, J. Chen, A. M. Rawlett, D.W. Price, J. M. Tour, Appl. Phys. Lett. 2001, 78 3735; b) Y. Chen, G. Y. Jung, D. A. A. Ohlberg, X. M. Li, D. R. Stewart, J. O. Jeppesen, K A. Nielsen, J. F. Stoddart, R. S. Williams, Nanotechnology 2003, 14, 462.

[9] E. Lörtscher, H. B. Weber, H. Riel, Phys. Rev. Lett. 2007, 98, 176807.

[10] E. Lörtscher, M. Elbing, M. Tschudy, C. von Hänisch, H. B. Weber, M. Mayor, H. Riel, Chem. Phys. Chem. 2008, 15, 2252.

[11] a) E. Lörtscher, J. M. Tour, J. W. Ciszek, H. Riel, Small 2006, 67, 50; b) E. Lörtscher, J. M. Tour, J. W. Ciszek, H. Riel, J. Phys. 2007, 61, 987; c) E. Lörtscher, H. Riel, ERCIM, 2006, 67, 50.

[12] a) C. J. Muller, J.M. van Ruitenbeek, L. J. deJongh, Physica (Amsterdam), 1992, C191, 485; b) J. Moreland, J.W. Elkin, J. Appl. Phys. 1985, 58,3888

[13] H. B. Weber, J. Reichert, F. Weigend, F. Ochs, D. Beckmann, M. Mayor, R. Ahlrichs, H. v. Löhneysen. Chem. Phys. 2002, 281, 113.

[14] M. Reed, C. Zhou, C. Muller, T. Burgin, J. Tour, Science 1997, 278, 252

[15] a) S. Yaliraki, A. Roitberg, C. Gonzalez, V. Mujica, M. Ratner, J. Chem. Phys. 1999, 111 , 6997; b) H. Gronbeck, A. Curioni, W. Andreoni, I. Am. Chem. Soc. 2000, 122, 3839; c) K. Stokbro, J. Taylor, M. Brandbyge, J. Mozos, P. Ordejón. Comput. Mater. Sci. 2003, 27, 151

[16] a) W. Wang, L. Takhee, M. A. Reed, Phys. Rev. B. 2003, 68, 035416; b) Z. Donhauser, D. Price, J. Tour, P. Weiss; J. Amer. Chem. Soc. 2003, $125,11462$.

[17] a) S. Hong, R. Reifenberger, W. Tian, S. Datta, J. I. Henderson, C. P. Kubiak, Superlattices Microstruct. 2000, 28, 289; b) D. Vonlanthen, A. Mishchenko, M. Elbing, M. Neuburger, T. Wandlowski, M. Mayor, Angew. Chem. 2009, $48,1$.

[18] M. Ruben, A. Landa, E. Lörtscher, H. Riel, M. Mayor, H. Görls, H. B. Weber, A. Arnold, E. Evers, Small, 2008, 4, 2229.

[19] A. S. Blum, J. G. Kushmerick, D. P. Long, C H. Patterson, J. C. Yang, J. C. Henderson, Y. X Yao, J. M. Tour, R. Sashidhar, B. R. Ratna, Nat. Mater. 2005, 4, 167. 